\section{Detecting Protein-Protein Interactions Using Renilla Luciferase Fusion Proteins}

BioTechniques 33:1044-1050 (November 2002)

\begin{abstract}
We have developed a novel system designated the luciferase assay for protein detection (LAPD) to study protein-protein interactions. This method involves two protein fusions, a soluble reporter fusion and a fusion for immobilizing the target protein. The soluble reporter is an N-terminal Renilla luciferase fusion protein that exhibits high Renilla luciferase activity. Crude cleared lysates from transfected Cos 1 cells that express the Renilla luciferase fusion protein can be used in binding assays with immobilized target proteins. Following incubation and washing, target-bound Renilla $l u$ -
\end{abstract}

ciferase fusion proteins produce light from the coelenterazine substrate, indicating an interaction between the two proteins of interest. As proof of the principle, we reproduced known, transient protein-protein interactions between the Cdc42 GTPase and its effector proteins. GTPase Renilla fusion proteins produced in Cos 1 cells were tested with immobilized recombinant GST-N-WASP and CEP5 effector proteins. Using this assay, we could detect specific interactions of Cdc42 with these effector proteins in approximately $50 \mathrm{~min}$. The specificity of these interactions was demonstrated by showing that they were GTPase-specific and GTP-dependent and not seen with other unrelated target proteins. These results suggest that the LAPD method, which is both rapid and sensitive, may have research and practical applications.

\section{INTRODUCTION}

Protein-protein interactions occur in all biological processes (15). In signal transduction pathways, these interactions transmit signals that regulate cell morphology, cell growth, apoptosis, and transcription. A variety of standard techniques is currently available for studying these interactions; however, most of these assays are time consuming and involve labeling at least one component of the assay with radioactivity, antibodies, and/or reporter molecules (15). For example, affinity chromatography, co-immunoprecipitation, Western blot analysis, and filter binding assays are commonly used to detect protein-protein interactions. The yeast two-hybrid assay is extremely useful in discovering new interacting proteins but is less useful for testing known interactions. Other methods such as detecting altered surface plasmon resonance energy require relatively expensive instrumentation (15). These methods, which often can detect only long-lived protein-protein interac- 
tions, usually are unable to detect directly or provide molecular-level information about the activated signaling intermediates that are generated via conformation-induced steric changes.

The Renilla reniformis luciferase gene expresses well in mammalian cells (11) and is commonly used as a control co-reporter with firefly luciferase constructs (5). Furthermore, Renilla luciferase and firefly luciferase have been used to study protein-protein interactions. For example, methods using fusion proteins of both Renilla luciferase and GFP can detect protein interactions using a modified fluorescence energy transfer method (20-22). Additional methods for using firefly luciferase include conjugation to protein-A (2), luciferase fusion proteins with biotin acceptor peptides (19), and a split luciferase based on RNA splicing (14). Here we describe a novel protein fusion system based on Renilla luciferase for studying protein-protein-interactions. This simple two-component binding assay, designated the luciferase assay for protein detection (LAPD), is rapid and sensitive. We have demonstrated the utility of this assay by examining interactions between the Cdc42 GTPase and its effector proteins.

\section{MATERIALS AND METHODS}

\section{Construction of Renilla Luciferase Fusion Proteins}

The sea pansy $R$. reniformis luciferase cDNA was amplified by PCR. Two linker primers, 5'-GAGGAATTCACTTCGAAAGTTTATGAT-3' and 5'-GAGGTCGACTTGTTCATTTTTGAGAAC-3', containing EcoRI and SalI sites, respectively, were used in PCR with a

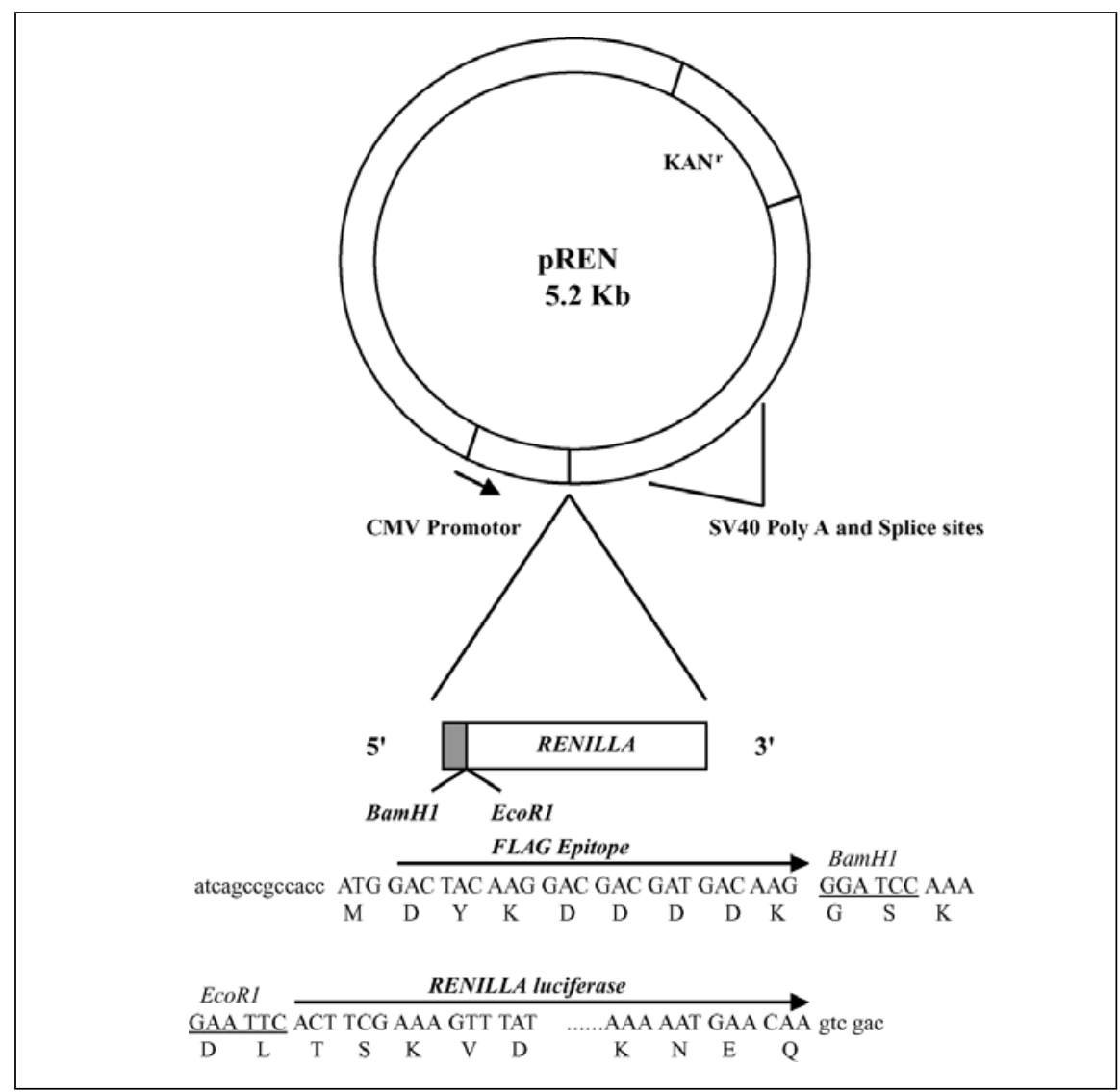

Figure 1. Structure of the pREN mammalian expression vector. Features indicated are the pCMV (CMV immediate early enhancer promoter), Renilla luciferase, and simian virus 40 splice site and polyadenylation sequence. The start methonine and FLAG epitope tag are denoted by the dark box. The nucleotide sequence and reading frame for generating $\mathrm{N}$-terminal fusion proteins with Renilla luciferase are shown. The cDNAs of interest are cloned as BamHI-EcoRI fragments in frame, with both the upstream FLAG epitope and downstream Renilla luciferase enzyme.

cytomegalovirus (CMV)-Renilla luciferase plasmid as a template (Promega, Madison, WI, USA). After amplification and EcoRI/SalI digestion, the 890-bp Renilla luciferase DNA fragment was subcloned into the EcoRI/XhoI site of pCAF2, a CMV mammalian epitopetagged expression vector (7). The resulting vector, pREN, was used as the parental vector for all additional constructs.

Previously described plasmids of Cdc42-L61 (9), Cdc42-N17 (13), and RhoA-L61 (1) were used as templates for PCR to generate cDNAs for these mutant GTPases. DNA encoding amino acids 2-193 of the Rho mutant was amplified with BamHI and EcoRI linker primers 5'-GAGGGATCCGCTGCCATCCGGAAGAAA- $3^{\prime}$ and $5^{\prime}$-GAGGAATTCCAAGACAAGGCAACCAGA-3'. DNA encoding amino acids $2-177$ of the Cdc42 mutants were amplified with BamHI and EcoRI linker primers 5'GAGGGATCCCAGACAATTAAGTGTGTTG-3' and 5'-GAGGAATTCGAGGGCAGCTAGGATAGC- $3^{\prime}$. The PCR products were then digested with BamHI and EcoRI restriction enzymes and cloned in frame into the BamHI/EcoRI site of pREN between the FLAG ${ }^{\circledR}$ epitope tag and Renilla luciferase (Figure $1)$. The integrity of each of these constructs was verified by DNA sequencing.

\section{Bacterially Produced GST Fusion Proteins}

Recombinant GST, GST-N-WASP, a fragment of N-WASP containing amino acids 151-277 (17), and GST-CEP5 (7) were produced using the pGEX-4T3 bacterial expression vector (Amersham Biosciences, Piscataway, NJ, USA), purified, and left immobilized on glutathione-agarose beads in buffer A (50 $\mathrm{mM}$ Tris, $\mathrm{pH} 7.5,50 \mathrm{mM} \mathrm{NaCl}, 5 \mathrm{mM}$ $\mathrm{MgCl}_{2}$ ) as previously described (7). The amount of each protein immobilized to the glutathione-agarose beads was estimated by SDS-PAGE.

\section{Production of Recombinant Renilla Fusion Proteins in Cos1 Cells}

Cos 1 cells were grown in DMEM containing $10 \%$ FCS and were split to $60 \%$ confluency one day before transfection. The pREN expression vector constructs $(2 \mu \mathrm{g})$ were transfected into 
Cos 1 cells by FuGENE ${ }^{\mathrm{TM}} 6$ transfection reagent (Roche Applied Science, Indianapolis, IN, USA) as previously described (7). Forty-eight hours after transfection, the cells were washed once with PBS and harvested by scraping into $1.2 \mathrm{~mL}$ buffer A containing $0.5 \%$ Triton ${ }^{\circledR}$ X-100 and protease inhibitors (0.5 mM PMSF, $25 \mu \mathrm{g} / \mathrm{mL}$ aprotinin, 50 $\mu \mathrm{g} / \mathrm{mL}$ pepstatin, and $25 \mu \mathrm{g} / \mathrm{mL}$ leupeptin). After centrifugation at $13000 \times$ $g$ for $2 \mathrm{~min}$, the supernatants were collected and used directly to determine the total number of light units in each of the lysates from the transfected cells. Luciferase activity was measured essentially as described in the Dual Luciferase Reagent Kit (Promega), except that the steps involving the detection of firefly luciferase were omitted (16). Briefly, $100 \mu \mathrm{L}$ Stop and Glow $^{\circledR}$ buffer (Promega) and $2 \mu \mathrm{L}$ coelenterazine substrate were added to each $12 \times 75-\mathrm{mm}$ glass tube. Renilla luciferase assays were initiated by the addition of $5 \mu \mathrm{L}$ Renilla containing supernatant to the substrate. These mixtures were then vortex-mixed and immediately measured for light units with a model LB9501 Berthold luminometer (Berthold Technologies, Bad Wildbad, Germany).

\section{LAPD}

LAPD is simple to perform. Because of intrinsic GTP of the Renilla GTPase fusion proteins, the assays were performed fresh, within $1 \mathrm{~h}$ of harvesting the cell lysates. Extracts containing equal amounts of Renilla luciferase activity (1 million light units) of each of the fusion proteins were added to $4 \mu \mathrm{g}$ immobilized GST, GST-N-WASP, or GST-CEP5 fusion proteins in buffer A containing $0.5 \%$ Triton X-100 and protease inhibitors in a final volume of 100 $\mu \mathrm{L}$. After a $20-\mathrm{min}$ incubation on ice, unbound and loosely bound proteins were removed by washing the beads four times in the same buffer, followed by centrifugation at $13000 \times g$. Approximately $70 \%, 16 \%, 2.5 \%$, and $2.2 \%$, respectively, of the total unbound Renilla activity was removed after the first, second, third, and fourth washes, respectively. After the fourth wash, the beads and associated proteins were resuspended in $30 \mu \mathrm{L}$ buffer $\mathrm{A}$. The amount of Renilla luciferase bound to the beads was determined by adding $15 \mu \mathrm{L}$ bead suspension to $100 \mu \mathrm{L}$ Stop and Glow buffer containing $2 \mu \mathrm{L}$ coelenterazine substrate and immediately measuring the light units.

\section{RESULTS}

\section{N-terminal Fusion Proteins with Renilla Luciferase Activity}

A cDNA-encoding Renilla lucife- 
rase was amplified by PCR and cloned into the EcoRI/XhoI site of a CMV mammalian expression vector. The resulting vector, pREN, contains, starting from the $5^{\prime}$-end, a Kozak consensus start methionine sequence, an N-terminal FLAG epitope, unique BamHI and EcoRI restriction sites, and the Renilla luciferase enzyme (Figure 1). Cos1 cells transfected with pREN could also be detected with immunofluorescence by the epitope tag (data not shown). Forty-eight hours after transfection, the cells were harvested and lysed by scraping in buffer containing $50 \mathrm{mM}$ Tris, $\mathrm{pH} 7.5,50 \mathrm{mM} \mathrm{NaCl}, 5 \mathrm{mM}$ $\mathrm{MgCl}_{2}$, and $0.5 \%$ Triton X-100. Transfection with $2 \mu \mathrm{g}$ pREN vector typically yielded extracts containing $2-8 \times 10^{9}$ light units $/ 100 \mathrm{~mm}^{2}$ plate of Cos 1 cells.

DNA encoding three mutant GTPases, constitutively active Cdc42-L61, dominant negative Cdc42-N17, and constitutively active Rho-L63 mutants were cloned in frame between the BamHI and EcoRI sites of the pREN vector (Figure 1). The corresponding plasmids were transfected into Cos 1 cells, and the fusion proteins were harvested 48 hours later. Using this protocol, all three mutant GTPase-Renilla fusion proteins had robust luciferase activity that was similar to the pREN control vector. Additional plasmid constructs with other Nterminal Renilla fusion proteins, including a fragment of B2 cyclin, also produced active luciferase fusion pro-

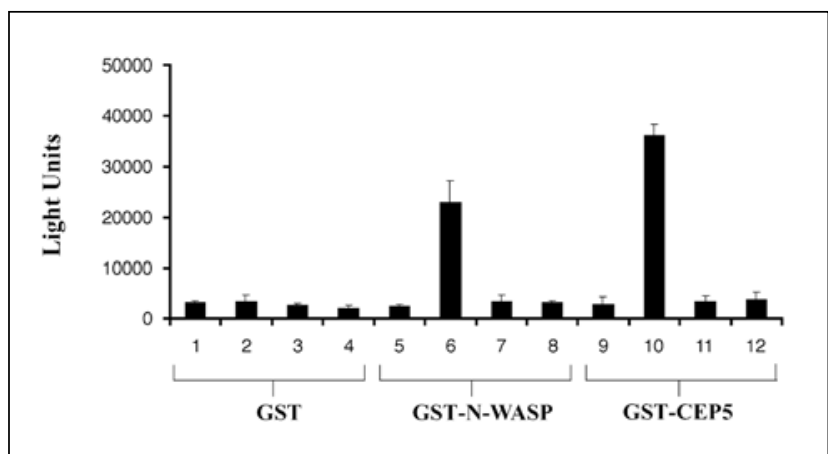

Figure 2. Detection of Cdc42 GTPase interactions with GST-N-WASP and GST-CEP5 by LAPD. Lysates from Cos 1 cells expressing Renilla (lanes 1, 5, and 9), Cdc42-L61-Renilla (lanes 2, 6, and 10), Cdc42-N17Renilla (lanes 3, 7, and 11), or RhoA-L63-Renilla (lanes 4, 8, and 12) was prepared $48 \mathrm{~h}$ after transfection. Equal amounts (1 million light units) of luciferase activity from each of the different lysates were added to the immobilized GST, GST-N-WASP, or GST-CEP5. Following a 20-min incubation, the beads were washed four times, and then an aliquot was added to the coelenterazine substrate and measured for light units. The mean of three independent experiments is represented. Error bars indicate standard deviation. teins with roughly similar levels of luciferase activity (data not shown). These results suggest that Renilla luciferase fusion proteins can maintain most of their luciferase enzymatic activity if N-terminal fusions are used as described here.

\section{LAPD}

Next, we tested whether these Renilla fusion proteins could be used to detect protein-protein interactions. A twocomponent binding assay (LAPD) was evised in which the binding of a soluarget was detected by measuring luciferase activity. Briefly, the cell beads. After incubation and washing, the protein-protein interaction was detected by the ability of the bound Renilla fusion protein to produce chemiluminescence after the addition of a luciferase substrate. In these assays, the amount of light produced was proportional to the amount of soluble fusion protein bound to the glutathione beads previous studies have shown that Renilla luciferase shows a linear range of luciferase activity over seven ders of protein concentration (16). ether it could detect the GTP-dependent interaction of Cdc42 with its effector proteins, N-WASP (12) and CEP5 (7). GST, GST-NWASP, and GSTCEP5 fusion proteins were produced in bacteria and left immobilized to the glutathione-agarose beads after purification. Lysates from the four Renilla fusion proteins were measured for luciferase activity, and an equal amount of light units (1 million) from each of the lysates was added to $4 \mu \mathrm{g}$ GST, GST-N-WASP, or GST-CEP5 immobilized beads. Following incubation for 20 min and four washes, a $15-\mu \mathrm{L}$ suspension of the residual beads was then added to the coelenterazine substrate, and the light units were measured. Under these experimental conditions, we found that the Renilla control protein did not bind to either GST or GST-N-WASP (Figure 2). In contrast, Cdc42-L61-Renilla fusion proteins bound to both GSTN-WASP and GST-CEP5 but not to GST (Figure 2). We also tested a dominant negative mutant of $\mathrm{Cdc} 42$ (Cdc42N17) that should not interact with GSTN-WASP or GST-CEP5 because it does not bind GTP. As expected, no significant binding of the dominant negative Cdc42-N17-Renilla was found with GST, GST-N-WASP, or GST-CEP5 (Figure 2). Rho-L63-Renilla fusion extracts also did not show GST, GST-NWASP, or GST-CEP5 binding (Figure 2). Collectively, these results show that LAPD can easily reproduce known protein-protein interactions between $\mathrm{Cdc} 42$ and its effector proteins.

\section{DISCUSSION}

In summary, we have developed LAPD, a simple, rapid method to detect protein-protein interactions using $R e$ nilla luciferase fusion proteins as the detection system. If these fusion proteins bind immobilized target proteins, then a larger visible signal is generated following the addition of a luciferase chemiluminescence substrate. Note that LAPD is rapid and that qualitative protein-protein interactions can be detected or excluded in less than 50 min once both sets of the recombinant proteins are available. Renilla luciferase is an ideal reporter for LAPD because it is small (36 kDa), highly sensitive (10), and retains most of its activity as a fusion protein while allowing the other component of the fusion to also remain biologically active. Based on these characteristics, we used Renilla fusion proteins to reproduce known proteinprotein interactions. Several additional aspects of this assay system should be noted. First, the ease, sensitivity, and rapidity of the assay depend on the remarkable activity of the Renilla luciferase reporter enzyme so that as little as $0.001 \mathrm{pg}$ can be detected (10). Second, by fusing the Renilla reporter into 
the C-terminus of probe proteins, almost all of the recombinant molecules are labeled. It is also likely that N-terminal fusion proteins may also be active and useful in the LAPD system. Third, LAPD offers the advantage of rapidly detecting protein-protein interactions without any additional amplification steps or the use of fluorescence or radioactivity. Finally, as with any assay, LAPD requires some optimization to determine the conditions that maximize detection sensitivity.

The sensitivity of LAPD for detecting the GTP-dependent interaction of Cdc42 with N-WASP was comparable to standard techniques such as radioactive filter binding assays and GST-capture experiments with these GTPases $(3,6)$. While the affinity of interactions between Cdc42-GTP and CRIB-containing effector proteins have a $\mathrm{K}_{\mathrm{d}}$ in the range of $4-0.8 \mu \mathrm{M}(18,24)$, it should be noted that under these conditions only a small percentage of the input Cdc42-L61-Renilla (2\%-3\%) bound to the immobilized GST-NWASP or GST-CEP5. The reason for this low binding is the fact that these interactions are dependent on GTP-bound Cdc42. Consistent with this idea, additional experiments that have revealed that prolonged incubation of the extracts (more than $3 \mathrm{~h}$ ) show decreased binding activity with the effector proteins, presumably due to GTP-hydrolysis becauuse no alteration in total Renilla luciferase activity was observed (unpublished data). In light of this, we expect the LAPD system might be useful in studying the activity of GTPase regulatory proteins and signaling pathways that alter the levels of GTP-bound GTPases. Furthermore, the LAPD system is likely to be suitable for the detection of other types of either stable or transient protein-protein interactions and might be simplified by producing both fusion proteins in bacteria rather than in mammalian cells, which would provide greater amounts of components that could be more easily standardized. It is already known that Renilla fusion proteins produced in bacteria retain their luciferase activity (Reference 8 and unpublished data). The LAPD system may be useful for drug discovery by facilitating high-throughput screens for agents that block known protein- protein interactions of medical interest. Finally, the recent ability to express (23) and optically image Renilla luciferase in live animals (4) may present the possibility of using variations of the LAPD system to study protein-protein interactions in vivo.

\section{REFERENCES}

1.Aspenstrom, P. and M.F. Olson. 1995. Yeast two-hybrid system to detect protein-protein interactions with Rho GTPases. Methods. Enzymol. 256:228-241.

2.Beigi, R., E. Kobatake, M. Aizawa, and G.R. Dubyak. 1999. Detection of local ATP release from activated platelets using cell surface-attached firefly luciferase. Amer. J. Physiol. 276:267-278.

3.Benard, V., B.P. Bohl, and G.M. Bokoch. 1999. Characterization of rac and cdc42 activation in chemoattractant-stimulated human neutrophils using a novel assay for active GTPases. J. Biol. Chem. 274:13198-13204.

4.Bhaumik, S. and S.S. Gambhir. 2002. Optical imaging of Renilla luciferase reporter gene expression in living cells. Proc. Natl. Acad. Sci. USA 99:377-382.

5.Bronstein, I., J. Fortin, P.E. Stanley, G.S. Stewart, and L.J. Kricka. 1994. Chemiluminescent and bioluminescent reporter gene assays. Anal. Biochem. 219:169-181.

6.Burbelo, P.D., D. Drechsel, and A. Hall. 1995. A conserved binding motif defines numerous candidate target proteins for both Cdc42 and Rac GTPases. J. Biol. Chem. 270:29071-29074.

7.Hirsch, D.S., D.M. Pirone, and P.D. Burbelo. 2000 . A new family of Cdc42 effector proteins, CEPs, function in fibroblast and epithelial cell shape changes. J. Biol. Chem. 275:22650-22656.

8.Jubin, R. and M.G. Murray. 1998. Activity screening of bacteria containing Renilla luciferase plasmids. BioTechniques 24:185-188.

9.Lamarche, N., N. Tapon, L. Stowers, P.D. Burbelo, P. Aspenstrom, T. Bridges, J. Chant, and A. Hall. 1996. Rac and Cdc42 induce actin polymerization and G1 cell cycle progression independently of $\mathrm{p} 65^{\mathrm{PAK}}$ and JNK/SAPK MAP kinase cascade. Cell 87:519-529.

10.Lorenz, W.W., R.O. McCann, M. Longiaru, and M.J. Cormier. 1991. Isolation and expression of a cDNA encoding Renilla reniformis luciferase. Proc. Natl. Acad. Sci. USA 88:4438-4442.

11.Lorenz, W.W., M.J. Cormier, D.J. O'Kane, D. Hua, A.A. Escher, and A.A. Szalay. 1996. Expression of Renilla reniformis luciferase gene in mammalian cells. J. Biolumin. Chemilumin. 11:31-37.

12.Miki, H., T. Sasaki, Y. Takai, and T. Takenawa. 1998. Induction of filopodium formation by a WASP-related actin-depolymerizing protein N-WASP. Nature 391:93-96.

13.Olsen, M.F., A. Ashworth, and A. Hall. 1995. An essential role for Rho, Rac, and Cdc42 GTPases in cell cycle progression through G1. Science 269:1270-1272.

14.Ozawa, T., A. Kaihara, M. Sato, K. Tachihara, and Y. Umezawa. 2001. Split luciferase as an optical probe for detecting protein-protein interactions in mammalian cells based on protein splicing. Anal. Chem. 73:2516-2521

15.Phizicky, E.M. and S. Fields. 1995. Proteinprotein interactions: methods for detection and analysis. Microbiol. Rev. 59:94-123.

16.Promega. 1998. Dual-Luciferase Reporter Assay System, p. 2-4. Technical Manual, Promega, Madison, WI.

17.Rohatgi, R., H.Y. Ho, and M.W. Kirschner. 2000. Mechanism of N-WASP activation by Cdc42 and phosphatidylinositol-4,5 bisphosphate. J. Cell Biol. 150:1299-1310.

18.Rudolph, M.G., P. Bayer, A. Abo, J. Kuhlmann, I.R. Vetter, and A. Wittiinghofer. 1998. The Cdc42/Rac interactive binding region motif of the Wiskott Aldrich syndrome protein is necessary but not sufficient for tight binding to $\mathrm{Cdc} 42$ and structure formation. J. Biol. Chem. 273:18067-18076.

19.Tatsumi, H., S. Fukuda, M. Kikuchi, and Y. Koyama. 1996. Construction of biotinylated firefly luciferases using biotin acceptor peptides. Anal. Biochem. 243:176-180.

20.Wang, Y., G. Wang, D.J. O'Kane, and A.A. Szalay. 1997. The Renilla luciferase-modified GFP fusion protein is functional in transformed cells, p. 419-422. In J.W. Hastings, L.J. Kricka, and P.E. Stanley (Eds.), Bioluminescence and Chemiluminescence: Molecular Reporting with Photons. John Wiley \& Sons, Chichester, UK.

21.Wang, Y, G. Wang, D.J. O'Kane, and A.A. Szalay. 2001. A study of protein-protein interactions in living cells using luminescence resonance energy transfer (LRET) from Renilla luciferase to Aequorea GFP. Mol. Gen. 264:578-587.

22.Xu, Y., D.W. Piston, and C.H. Johnson. 1999. A bioluminescence resonance energy transfer (BRET) system: application to interacting circadian clock proteins. Proc. Natl. Acad. Sci. USA 96:151-156.

23.Yu, Y.A., S. Caltharp, and A.A. Szalay. 2002. Inducible gene expression in vivo using a Renilla luciferase-GFP fusion construct, $\mathrm{p}$. 465-468. In J.F. Case, P.J. Herring, B.H Robinson, S.H.D. Haddock, L.J. Kricka, and P.E. Stanley (Eds.), Proceeding of the 11th International Symposium on Bioluminescence and Chemiluminescence. John Wiley \& Sons, Chichester, UK.

24.Zheng, B., Z.X. Wang, and Y. Zheng. 1997. Characterization of the interactions between the small GTPase Cdc42 and its GTPase-activating proteins and putative effectors. Comparison of kinetic properties of $\mathrm{Cdc} 42$ binding to the Cdc42-interactive domains. J. Biol. Chem. 272:1999-2007.

This work was supported by grant no. DAMD17-99-1-9190 from the Department of Defense Breast Cancer Program to P.D.B. Address correspondence to Dr. Peter D. Burbelo, Lombardi Cancer Center and the Department of Biochemistry and Mole- 
cular Biology, Georgetown University Medical Center, Washington, D.C. 20057, USA. e-mail: burbelpd@georgetown.edu

Received 15 May 2002; accepted 16 August 2002.

\section{Peter D. Burbelo, Adam \\ E. Kisailus, and Jeremy \\ W. Peck \\ Georgetown University Medical \\ Center \\ Washington, D.C., USA}

For reprints of this or any other article, contact Reprints@BioTechniques.com

\section{Negative Purification Method for the Selection of Specific Antibodies from Polyclonal Antisera}

BioTechniques 33:1050-1054 (November 2002)

\begin{abstract}
We developed a protocol to remove nonspecific antibodies from polyclonal antisera by adsorption on non-target antigens immobilized on nitrocellulose membranes. This "negative" purification method is simple and provides better immunoreagents than the blocking of nonspecific antibodies in solution or the enrichment of specific antibodies on nitrocellulose membranes. For routine applications, this method is quicker and cheaper than the purification protocols based on selective precipitations and affinity chromatography.
\end{abstract}

\section{INTRODUCTION}

Antibodies are instrumental in protocols for immunolocalization, immunopurification, Western blot analysis, EMSA super-shifts, or the screening of cDNA expression libraries - not to mention their use in detection kits. Each of these applications has their own requirements in terms of the purity of the antibodies. Although monoclonal antibodies are free from interfering host immunoglobulins, their production is expensive and requires technical knowledge and animal cell culture facilities. Alternatives such as the phage display technology and the in vitro production of recombinant antibodies suffer from similar limitations.

Thus, the use of polyclonal antisera remains very popular but necessitates the removal of nonspecific antibodies. Purification typically begins with a combination of ammonium sulfate/ caprylic acid precipitation or ammonium sulfate/DEAE chromatography (5), and nearly pure antibodies are recovered, although frequently at low titers because of their limited solubilization or inactivation (2). They are typically submitted to an additional affinity pu- rification. Protein A, protein G, or antiIgG affinity chromatography of $\mathrm{IgG}$ from ascites or polyclonal antisera (1) is a straightforward method but not always suitable for all species and antibody subclasses (4), and nonspecific IgG antibodies still end up in the final eluate.

Antigen affinity chromatography is the best technique to obtain highly purified and specific antibodies (3) but often requires harsh conditions (e.g., extreme $\mathrm{pH}$, chaotropic and denaturating agents, etc.) to elute the antigen, causing a loss of activity (2,3). Finally, whichever protocol is used, it has to be optimized for each antigen, which is hardly conceivable for the routine production of sera against a significant number of the proteins present on a 2-D electrophoresis gel.

Quicker protocols have been used to obtain partially purified preparations. These consist simply of the centrifugation of the nonspecific antibodies inhibited by blocking mixtures (8) or of the adsorption of specific antibodies on nitrocellulose-bound antigens and their subsequent recovery (7).

Here we discuss what we call a negative antibody purification in which we remove nonspecific antibodies by incubating polyclonal antisera with non-target proteins that are adsorbed on a membrane. The efficiency of each method is evaluated using Western blot analysis.

\section{MATERIALS AND METHODS}

\section{Bacterial Strains and Growth Conditions}

E. coli BL21 (DE3) ${ }^{\circledR}$ (Novagen, Madison, WI, USA) clones were grown in liquid $\mathrm{LB}$ medium at $37^{\circ} \mathrm{C}$ under constant agitation in the presence of 100 $\mu \mathrm{g} / \mathrm{mL}$ ampicillin. Two bacterial test clones were used. The pET15b-GMD clone contained the $\mathrm{pET} 15 \mathrm{~b}^{\circledR}$ plasmid (Novagen) with a 1122-bp NdeI/XhoI cDNA fragment of the Arabidopsis thaliana GDP-D-mannose-4,6-dehydratase gene (GMD) (GenBank ${ }^{\circledR}$ accession no. U81805) and the pET21c-GE clone contained the $\mathrm{pET} 21 \mathrm{c}^{\circledR}$ plasmid (Novagen) with a 1056-bp NotI/SalI cDNA fragment corresponding to the $A$. thaliana UDP-glucose-4-epimerase gene (GE) (accession no. Z54214). 